\title{
Facial Expression-Aware Face Frontalization
}

\author{
Yiming Wang ${ }^{1}$, Hui $\mathrm{Yu}^{1, \star}$, Junyu Dong ${ }^{2}$, Brett Stevens ${ }^{1}$, Honghai Liu ${ }^{1}$ \\ ${ }^{1}$ University of Portsmouth, Portsmouth, United Kingdom \\ ${ }^{2}$ Ocean University of China, Qingdao, China
}

\begin{abstract}
Face frontalization is a rising technique for view-invariant face analysis. It enables a non-frontal facial image to recover its general facial appearances to frontal view. A few pioneering works have been proposed very recently. However, face frontalization with detailed facial expression recovering is still very challenging due to the non-linear relationships between head-pose and expression variations. In this paper, we propose a novel facial expression-aware face frontalization method aiming at reconstructing the frontal view while maintaining vivid appearances with regards to facial expressions. First of all, we design multiple face shape models as the reference templates in order to fit in with various shape of facial expressions. Each template describes a set of typical facial actions referred to Facial Action Coding System (FACS). Then a template matching strategy is applied by measuring a weighted Chi Square error such that the input image can be matched with the most approximate template. Finally, Robust Statistical face Frontalization (RSF) method is employed for the task of frontal view recovery. This method is validated on a spontaneous facial expression database and the experimental results show that the proposed method outperforms the state-of-the-art methods.
\end{abstract}

\section{Introduction}

Facial expression recognition (FER) forms the essence of human-machine system, and therefore is one of the most active research topic in the field of humancomputer interaction, computer vision and machine intelligence $[1,2]$. For over two decades, much effort has been made to improve the FER system. However, most existing FER methods were still focusing on the recognition of frontal or near-frontal facial images with posed facial expression [3]. The performance of these methods will drop dramatically in the uncontrolled real-world environment, especially when there are large occlusion and head-pose variations. With the quick development of human-machine system, there has been a continuously increasing demand for effective spontaneous FER methods.

One of the key challenges for in-the-wild facial expression recognition is how to tackle the variations of out-of-plane head rotation. There are only a few approaches proposed for view-invariant FER. Up to now, these methods can be

\footnotetext{
* Corresponding author: Hui Yu, at School of Creative Technologies, University of Portsmouth, Portsmouth, United Kingdom. Tel.:(+44)23 92845470 Email address: hui.yu@port.ac.uk
} 
divided into two categories: view-based methods and dictionary learning methods. In [4], a typical multi-view approach is applied, in which multiple discrete yaw angles are predefined. For each face image, the head pose is estimated and matched to the closest predefined angle. Then a view-specific facial expression classifier is trained in each discrete angle. It is obvious that a pose estimation step must be performed first, and the whole system has to be trained per viewpoint/person/expression. Similar situation exists in [5] and [6]. In [6] and [7],two approaches of view normalization are described. Rudovic et al. [7] present a pairwise viewpoint normalization in which Coupled Gaussian Processes regression are used to model the pair-wise facial geometric features (facial points) between non-frontal face and its corresponding frontal counterpart. View normalization can tackle an input face image with unseen viewpoint, which is superior to pose-wise FER approaches. However, the pose estimation step is still inevitable. Furthermore, the pair-wise view modelling is time consuming and requires large amount of training data to ensure accuracy. For dictionary learning methods, include [8] and [9], both of them use SIFT [10] as low-level feature descriptor and encode SIFT feature pose-wise. Specifically, [8] uses generic sparse coding and [9] uses supervised super-vector encoding for high-level feature learning. After learning the high-level features from different views, a single classifier will be trained. Dictionary learning approaches still need to learn the features per viewpoint/person/expression in order to achieve promising results.

Apparently, current view-invariant FER methods highly rely on the quality of training data. These approaches require a large data volume in terms of different expressions and poses, and some of them even need to be trained person-specifically. The satisfied database is often not readily available.

As a newly rising research topic in view-invariant face analysis, face frontalization can overcome the drawbacks mentioned above. It aims to recover the frontal face from unconstrained image. Until now, the approaches and contributions in this field are very limited. The key idea of $2 \mathrm{D}$ face frontalization is estimating frontal facial shapes and compensating the missing part of frontal facial textures. Face frontalization starts from facial landmark localization, then performs shape matching and texture fitting schemes to reconstruct frontal face. Existing methods can be divided into two categories: 3D assisted methods and $2 \mathrm{D}$ methods. There are many 3D-assisted approaches that synthesize the 3D facial model from several images of one person and result in person-specific face frontalization $[11,12]$. These methods will need a large amount of facial images captured from poses and expressions. Furthermore, person-specific face frontalization approaches are often impractical since they cannot deal with a totally new face from a single image. Therefore, we will focus more on generic face frontalization. Hassner et al. [13] present a generic face frontalization method which attempt to approximate 3D facial shapes from a single 2D facial image. Given a single reference 3D surface, the landmarks of input image is projected to their 3D positions of reference 3D surface and compute a projection matrix. By generating frontal view using the estimated 3D surface, 3D coordinates will be projected back to the input image. The missing part due to pan angles of 
head pose is compensated by the corresponding symmetric parts of the face. This method is a breakthrough of generic face frontalization. However, the nonfrontal face with tilt angles cannot be recovered, and the symmetry-based face compensation is not always reasonable, especially when there are occlusions or non-symmetric facial expressions. In general, 3D assisted method can achieve high performance of face frontalization. But 3D facial shape estimation from 2D is a hard problem and may potentially lead to misalignment. There are only a few works that has been done on 2D methods. In [14], the face image is divided into several overlapping blocks and Markov Random Field is employed to optimize patch-based local warps based on an assumption that the mean value of intensity of each block is the same. The global frontal face can be predicted by optimizing each local warp. In [15], Sagonas at al. presents Robust Statistical face Frontalization (RSF) based on the fact that frontal facial images have a smaller value of nuclear norm when compared with non-frontal facial images. RSF firstly warps a non-frontal face into a reference frame (base mesh). Then an optimization problem is solved by iteratively minimizing the nuclear norm of warped image.

Compared with the traditional view-invariant FER methods that is sensitive to the quality of training data, 2D face frontalization methods is potentially a better alternative for FER in the wild. Considering that 3D assisted face frontalization approaches has many limitations, we mainly focus on 2D methods. Thus, the purpose of this work is to derive a generic facial expression-aware face frontalization which is robust to individual differences, occlusions and head pose variations in whatever pan and tilt angles.

No work has been done to apply face frontalization to view-invariant FER so far due to the problem of identity bias [2] in 2D FER task. It has been proved that facial features are always biased to identity-related interpretation but poor in describing facial expressions. Current $2 \mathrm{D}$ face frontalization methods tend to aggravate this problem since they always need a shape template to perform rigid shape matching. The expression-related information of both facial shapes and textures may be lost after shape matching. This situation, therefore, makes it more difficult to apply face frontalization to FER.

In this research, we present a novel view-invariant FER method based on face frontalization. Knowing that changes in non-rigid expression and changes in head pose in 2D cannot be linearly decomposed $[6,7]$, it is challenging perform a non-rigid frontal facial shape recovery from non-frontal images. Current 2D face frontalization approaches often present a rigid shape matching that matches the shape of input image with a single reference template, which often leads to a huge loss of facial expression cues. The non-linear problem mentioned above is very difficult to solve, but we can present a approximated solution when there are multiple templates instead of one single template, and non-frontal face shape can be matched with the most appropriate templates. Inspired by this idea, we developed a Facial Expression-Aware face Frontalization (FEAF) method using multi-template model. In this method, five different templates are developed. Among them, one face mesh is neutral and the other four are expression-enriched 
templates. These templates are designed by a combination of Action Units (AUs) described in Facial Action Coding System (FACS) according to the commonly accepted expert knowledge of the relations between six universal emotions and AUs [16].

With the five templates, a template matching step will be undertaken in order to match the input image with the most appropriate template. The landmarks of the input image must be detected first, but there is no need to detect all facial points. Only the landmarks located in the expression-enriched facial regions are needed. The satisfactory landmark detection method is Supervised Decent Method (SDM) [17] which can correctly localize 49 points in the facial regions of eyes, eyebrow, nose and mouth. Then the similarity between the input face shape and the five templates can be measured by using weighted Chi square error. After template matching, RSF is employed to fit the texture of input image with corresponding template. By observing that the running time of RSF can be significantly reduced if the initialization highly approximates to the optimal value, we use a landmark detector to localize facial landmarks and use its output as the initialization of RSF so that the efficiency can be improved prominently. Finally, some commonly used feature extraction methods and machine learning techniques will be employed to recognize the six universal facial emotions (happy, sad, surprise, fear, angry and disgust). The experimental validation shows that FEAF achieves an effective face frontalization with powerful description of facial expressions. FEAF outperforms the state-of-the-art methods for FER in the wild. The main contributions of this research include:

1). We develop a multi-template model for facial expression-aware face frontalization in which detailed facial expression appearances are effectively reconstructed. As far as we know, this research is the first of its kind to recover full frontal facial expressions from unconstrained facial images.

2 ). Whatever pan or tilt angles of head pose in test images (even the untrained ones) can be recovered in this work.

3). The efficiency of RSF is improved by providing an appropriate initialization.

\section{Multi-template Based Face Frontalization}

\subsection{Template design and template matching}

As previously discussed, 2D face frontalization methods may lose the expressionrelated cues during shape matching. Especially for RSF, the face shape will be arbitrarily warped to a neutral template whatever the shape of input image is. This warping strategy will cause a huge loss of expression-related information. In order to overcome this problem, we present a novel method by designing multiple templates which involve variant face shapes in terms of expressions, such that the non-frontal facial images can be warped to its most approximate template.

It is commonly accepted that the facial areas around eyebrow, eyes and mouth are the most enriched facial region related to facial emotions [18]. So we empirically design five templates which include five apparent characteristics: eyes wide 
open, eyebrow lower, lips apart, mouth wide open and neutral, as is exhibited in Fig.1. These facial behaviours are the most significant shape features in expressions of human beings.

The idea of the template design is inspired from FACS. FACS defines many AUs that encodes the basic unit of facial configurations. It is generally accepted that combination of different AUs can generate meaningful facial emotions. For example, the emotion of sad is often a combination of AU1, AU4 and AU15, meanwhile fear is usually a combination of AU1, AU2, AU4, AU5, AU7 and AU26. Apparently, Au1 and AU4 are basic component of both sad and fear. So we design the template 2 which includes AU1 and AU4 so that an input facial image with the emotion of sad or fear will be easier to be classified into t2. The other templates are designed in the similar way. Considering that many AUs, as well as their combinations, share the same shape and may not important in determine emotional categories, there is no need to design more templates. Five template are enough to meet the basic demand of expression-aware face frontalization.

Fig.1(a) means eyes wide open which is often related to surprise and fear. Fig.1(b) indicates lowering the eyebrow, which often appears in sad, angry and disgust. Fig.1(c) suggests lips apart that is relevant to smile and fear. Fig.1(d) means mouth wide open that often exists in surprise. Fig.1(e) is neutral face shape. As is shown in the figure, these five templates are very different from each other and can be immediately distinguished only by shapes. So the probability of mismatch can be reduced in the template matching step.

The procedure of of template design is as follow: (1) Given the training data, we manually select the frontal faces without occlusion. (2) Manually classifying the clean frontal face to five categories in terms of their most significant shape features. For those images that are suitable to more than one templates, a priority queue is listed, in which changes in eyebrow are priority, followed by eyes and finally the mouth. For example, a facial image with both eyes and mouth wide open will be assigned to template $\mathrm{t} 1$, but not $\mathrm{t} 4$, according to this priority list. (3) Five templates are obtained by computing the mean shape of each category.

Template matching will match the position of landmarks of input face with an appropriate template. It, thus, start from facial landmark detection. By observing that importance of different facial landmarks varies, there is no need to localize all these points. SDM is the best choice for this task due to its small computational cost and high accuracy in face alignment. It can effectively localize 49 fiducial landmark points in the region of eyebrow, nose, eyes and mouth. Then the facial points are normalized using Procrustes analysis.

Then, a similarity measure is undertaken to perform template matching. We select Chi square test statistic $\left(\chi^{2}\right)$ for similarity assessment. $\chi^{2}$ statistic is a test of goodness of fit. It evaluates how well a statistic model fits with sets of observations. In this application, it has been discussed that some landmark points contribute more than others regarding facial expression variations. Therefore, the facial landmarks are weighted based on the importance. The weighted $\chi$ 


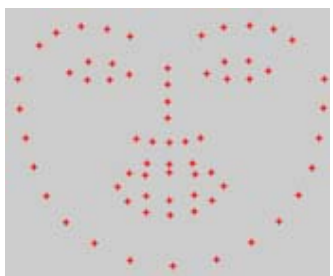

(a) t1

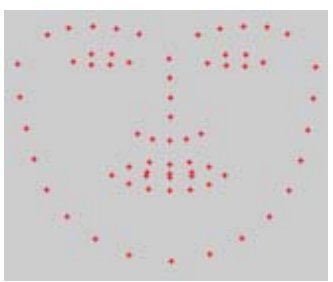

(b) $\mathrm{t} 2$

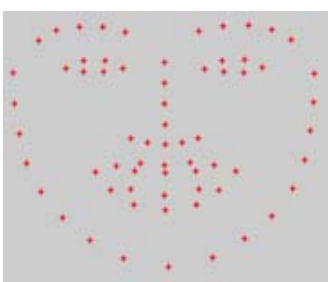

(c) t3

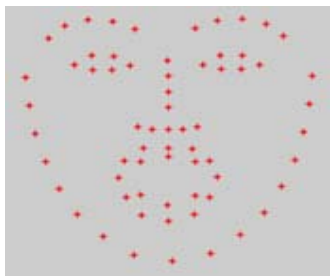

(d) t4

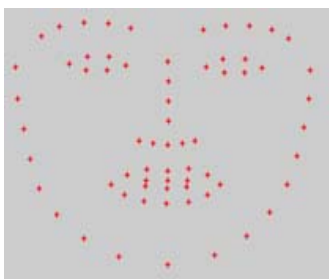

(e) t5

Fig. 1. Five templates of face shape

measure is then given as

$$
\chi_{w}^{2}(S, T)=\sum_{i, j} w_{j} \frac{\left(S_{j}-T_{i, j}\right)^{2}}{S_{j}+T_{i, j}}
$$

where $S$ and $T$ are the positions of landmarks of input image and template, respectively. $T_{i, j}$ means the $j$ th landmarks in template $t_{i}$. The function is weighted by $w$, which full considers the importance of the eyes and eyebrow. Fig.2 shows the weighting scheme.

\subsection{Improved RSF}

RSF is close related to Transform Invariant Low-rank Texture (TILT) [19]. RSF is based on the fact that frontal face image has the minimum rank (smallest value of nuclear norm) when compared to non-frontal face images. So a optimization problem can be described as follow:

$$
\begin{aligned}
& \underset{L, e, c, \Delta p}{\operatorname{argmax}}\|L\|_{*}+\lambda\|E\|_{1} \\
& \text { s.t. }\left\{\begin{array}{l}
H^{(1)}(\Delta p, c, e)=x(p)+J(p) \Delta p-U c-e=0 \\
H^{(2)}(L, c)=L-\sum_{i=1}^{k} R\left(u_{i}\right) c_{i}=0
\end{array}\right.
\end{aligned}
$$

where $L$ is low-rank matrix which is expected to be the recovered frontal face. $E$ is sparse error matrix. $X(p)$ is the warped image and $p$ is the parameter of its shape referred to the equation $s=s_{0}+U_{s} p$ defined by Active Shape Model 


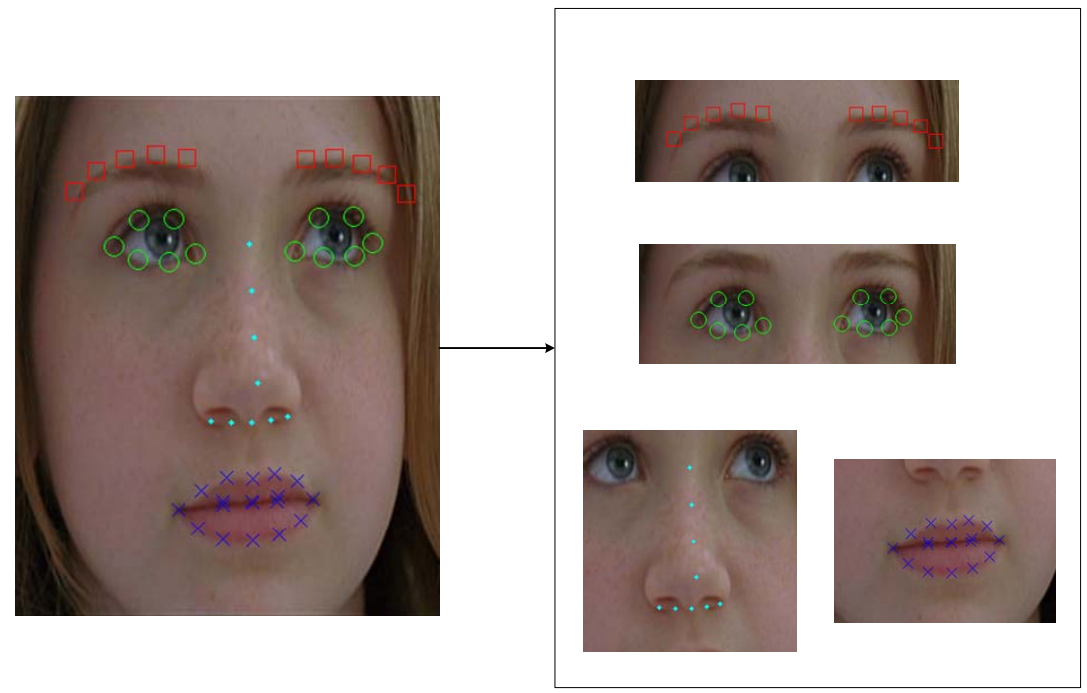

Fig. 2. Weighted Chi square measure where the weighted values are set by red ' $\square$ ' 2.0, 'O' 1.5, ' $\times$ ' 1.0 and '•' 0.0 .

(ASM) [20] where $s_{0}$ is the reference template. $J(p)=x(p) \frac{\partial W}{\partial p}$ is the Jacobian matrix. $U=\left[u_{1}\left|u_{2}\right| \cdots \mid u_{k}\right]$ is the pre-computed appearance model (eigen faces computed on only clean frontal faces). Equation $H^{(1)}$ indicates that the addition of low rank texture $L$ and sparse error $E$ agrees with the warped image, such that $X(p)=L+E$. In equation $H^{(2)}$, the low rank matrix is represented as a linear combination of $U$ where $c$ is its parameter. $R(\bullet)$ is an operator that reshape a vector to its corresponding matrix. By introducing augmented Lagrangian method (ALM) and alternating directions method of multipliers (ADMM). The parameters can be optimized iteratively.

The whole RSF includes outer loop and inner loop. Inner loop solves the above optimization problem and returns $\Delta p$. Outer loop updates $p$ by $p=p+\Delta p$ and then use the new parameter to compute warp image $X(p)$ and Jacobian matrix. Both variables and parameter $p$ will be inputs for the new round of inner loop.

In [15], the author does not provide the source code of RSF, so we implement it independently. One of the key techniques of RSF is image warping. It aims to warp the position of each pixel of input image to its corresponding location in base mesh (reference template). We employed piecewise affine warping method [21] for this task. Piecewise affine warping is based on an assumption that image warping on a small local region is linear although whole face warping is nonlinear. Given a base shape, Delaunay triangulation is used to create multiple non-overlapping triangles whose vertices are facial landmark points. Delaunay triangulation ensures that the circumcircle associated with each triangle contains no other points within it. Each triangle accounts for a fairly small 
region such that linear affine warping is reasonable. All these triangles make up the mesh.

For a pixel within triangle $\left(x_{i}^{0}, y_{i}^{0}\right),\left(x_{j}^{0}, y_{j}^{0}\right)$ and $\left(x_{k}^{0}, y_{k}^{0}\right)$ in base mesh, its location $\left(x^{0}, y^{0}\right)$ can be expressed as:

$$
\begin{aligned}
& \left(x^{0}, y^{0}\right)=\left(x_{i}^{0}, y_{i}^{0}\right)+\alpha\left[\left(x_{j}^{0}, y_{j}^{0}\right)-\left(x_{i}^{0}, y_{i}^{0}\right)\right]+\beta\left[\left(x_{k}^{0}, y_{k}^{0}\right)-\left(x_{i}^{0}, y_{i}^{0}\right)\right] \\
& \left\{\begin{array}{l}
\alpha=\frac{\left(x^{0}-x_{i}^{0}\right)\left(y_{k}^{0}-y_{i}^{0}\right)-\left(y^{0}-y_{i}^{0}\right)\left(x_{k}^{0}-x_{i}^{0}\right)}{\left(x_{j}^{0}-x_{i}^{0}\right)\left(y_{k}^{0}-y_{i}^{0}\right)-\left(y_{j}^{0}-y_{i}^{0}\right)\left(x_{k}^{0}-x_{i}^{0}\right)} \\
\beta=\frac{\left(y^{0}-y_{i}^{0}\right)\left(x_{j}^{0}-x_{i}^{0}\right)-\left(x^{0}-x_{i}^{0}\right)\left(y_{j}^{0}-y_{i}^{0}\right)}{\left(x_{j}^{0}-x_{i}^{0}\right)\left(y_{k}^{0}-y_{i}^{0}\right)-\left(y_{j}^{0}-y_{i}^{0}\right)\left(x_{k}^{0}-x_{i}^{0}\right)}
\end{array}\right.
\end{aligned}
$$

The warped image will follow the same deformation. Its position $(x, y)$ in triangle $\left(x_{i}, y_{i}\right),\left(x_{j}, y_{j}\right)$ and $\left(x_{k}, y_{k}\right)$ is given as:

$$
(x, y)=\left(x_{i}, y_{i}\right)+\alpha\left[\left(x_{j}, y_{j}\right)-\left(x_{i}, y_{i}\right)\right]+\beta\left[\left(x_{k}, y_{k}\right)-\left(x_{i}, y_{i}\right)\right]
$$

With the location of each pixel warped in the base mesh, the pixel value will be computed by using linearly interpolation.

The time complexity of RSF is relatively high. Both optimization in inner loop and piecewise affine warping in outer loop are time consuming. In practical, there will be more than 120 iterations in each inner loop and no less than 40 iterations in outer loop. This situation makes RSF computationally expensive.

Considering that RSF optimization is unsupervised local direction search, it will lead to very slow convergence and expensive time cost. If the initialization is localized near optimal value, the number of outer loop will drop significantly. In order to achieve the expected initialization, a accurate landmark detector is inevitable. SDM is adequate, but this time 66 points is needed to synthesize the face mesh instead of the original 49 points. The authors of SDM only provide a 49-points detection model and the training part is totally hidden. So we implement SDM and train the 66 -points landmark model. The 66 points computed using SDM is not so accurate compared with its 49-points counterpart, but it is still very close to the optimal values. In this strategy, outer loop of RSF can converge within five rounds. By introducing this simple step, this method become much more efficiency. The whole approach is shown in Algorithm 1.

\section{Experiment}

\subsection{Database and evaluation protocol}

We evaluate the proposed method on Statistical Facial Expression in the Wild (SFEW) [22]. SFEW contains 700 images with spontaneous facial expressions labelled by seven categories: six universal emotions and neutral. The images are captured from movies, which covers different real-world conditions such as occlusion, low resolution and variations in illumination and head pose. SFEW provide a clear evaluation protocol. Each category include 2 image sets: one for 


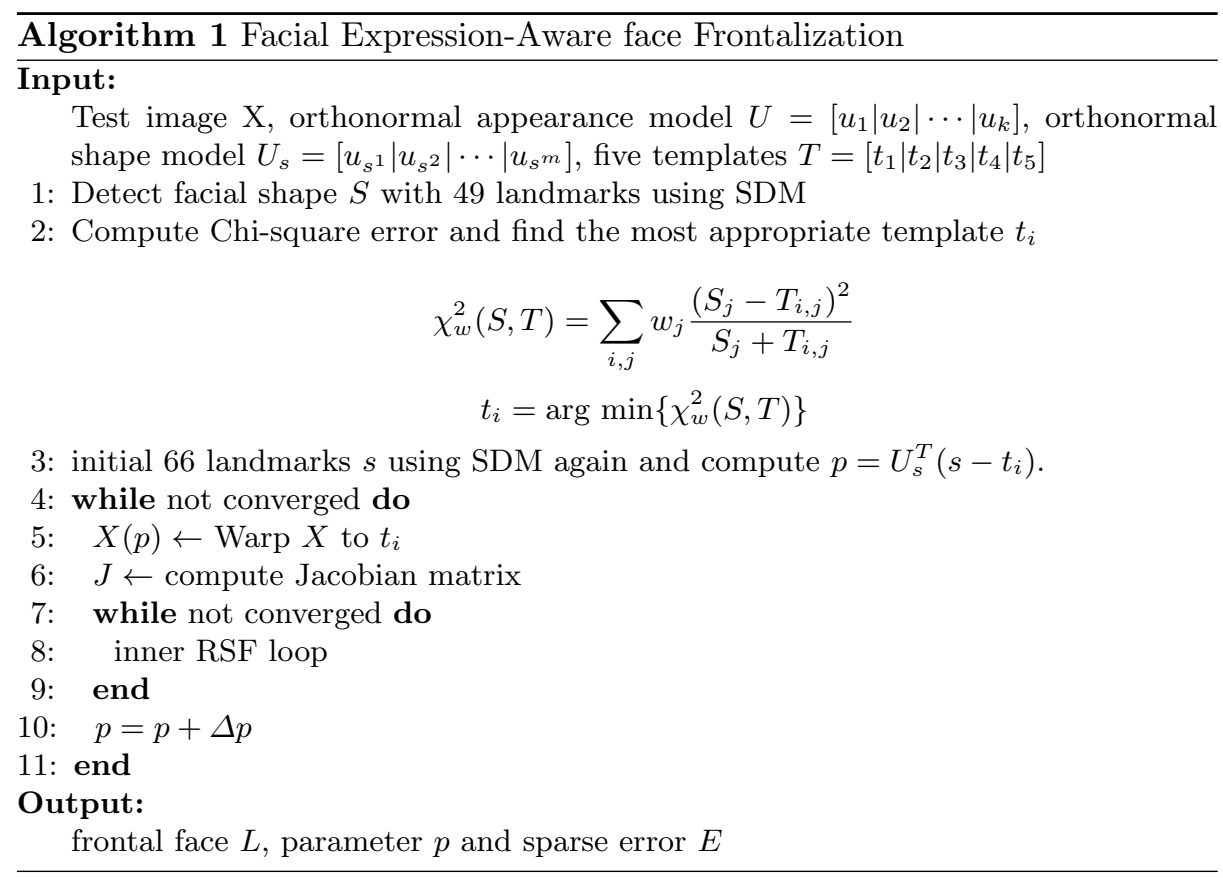

training and the other for testing. The experiment is strictly person-independent in which the images of one specific person with one specific emotion can only exist in one image set, whatever training or testing set, in order to evaluate the generalization performance on totally new faces.

We perform face frontalization on both training and testing data. Then the uniformed Local Binary Pattern (LBP) features are extracted and finally, Support Vector Machine (SVM) with Radial Basis Function (RBF) kernel is utilized for emotion classification.

\subsection{Face frontalization}

In this section, we will show visual results of face frontalization. In fig. 3 , the sub-figures visually display the face frontalization result on the emotions of angry, disgust, fear, happy, sad and surprise, respectively. It can be seen that various head-poses in pan and tilt angles are recovered to their frontal views. The robustness against occlusion can be demonstrated by observing that all the subjects who wear glasses can be amended. Meanwhile, some images with low resolution can be processed as well. Fig.3 shows the robustness of FEAF with regards to occlusion, low resolution and head-pose variations. More importantly, the displayed frontal faces maintain detailed information of facial expressions.

Fig. 4 shows the comparison of RSF and proposed method. We can intuitively see that both method achieve successful frontalization. But RSF lose much information regarding facia expressions. Whilst our method recovers the frontal view 

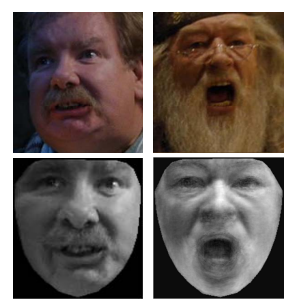

(a)
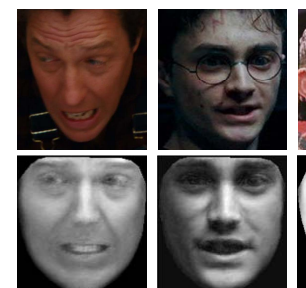

(c)
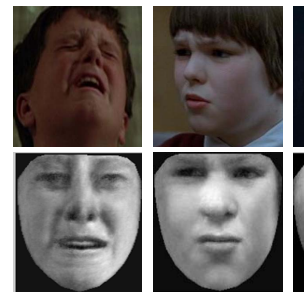

(e)

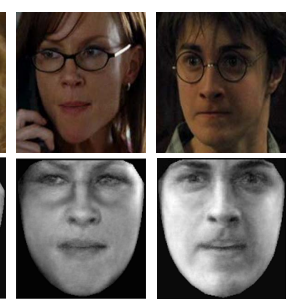

)

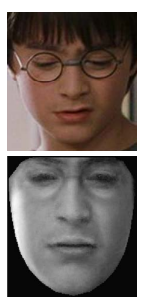

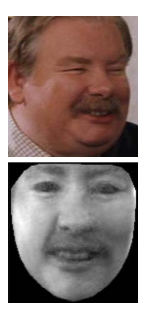

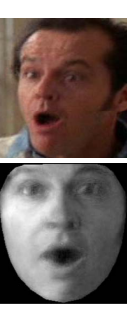

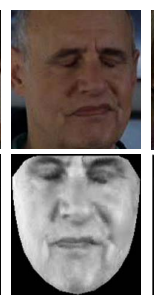

(b)
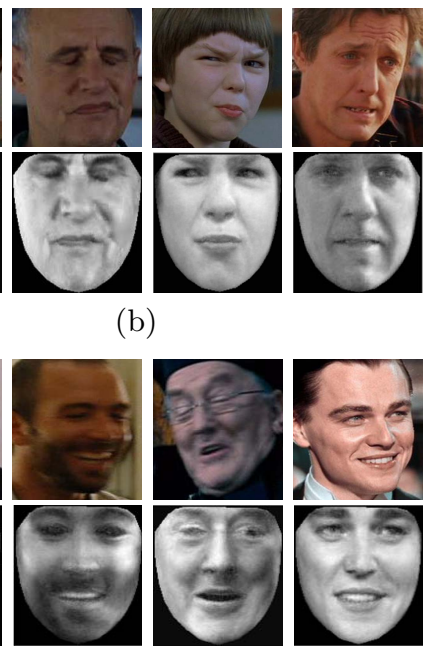

(d)

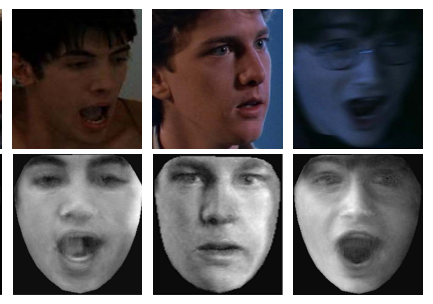

(f)

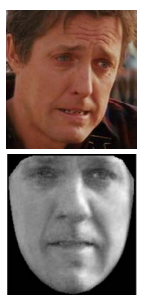

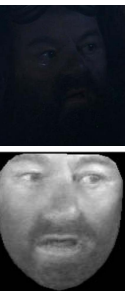

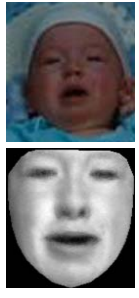

g. 3. Face frontalization

with more details of expressions. It is generally believed that texture cues are more important than shape cues in face analysis. But from this comparison, it is obvious that shape features are also important in synthesizing the frontal faces and FEAF has a superior performance in the maintenance of facial expressions.

Another application of FEAF is face alignment. The position of facial landmark points can be computed by $s=t_{i}+U_{s} p$ where $t_{i}$ is the shape of selected template and $p$ is the shape parameter obtained by the output of our method. Landmark detection is not the main task of this work, but it still achieves considerable results, shown in Fig.5.

\subsection{Facial expression recognition in the wild}

The FER performance of FEAF is evaluated on SFEW database. With the derived frontal faces, we normalize the pixel values to the range of $0 \sim 255$ and extract LBP features [23]. Each frontal face image is divided into 8 by 7 overlapping blocks with $70 \%$ overlapping rate and a spatially enhanced LBP feature representation is obtained by concatenating the histogram computed from each 

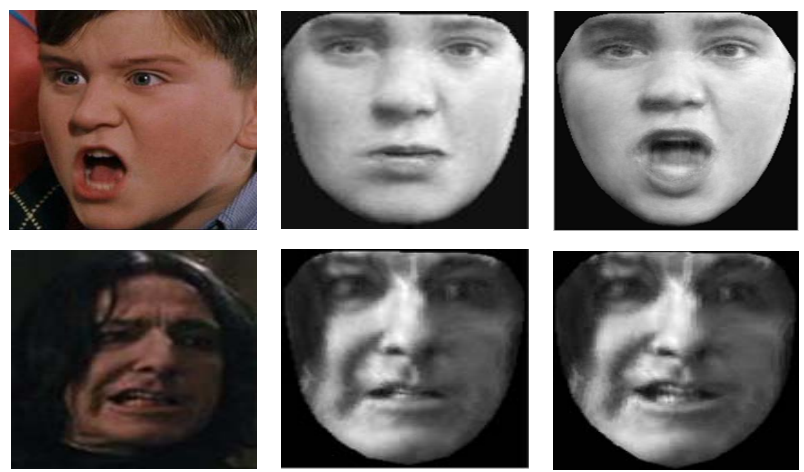

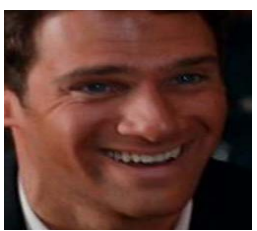

(a) Original image

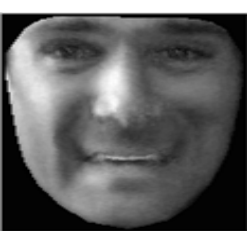

(b) RSF warp

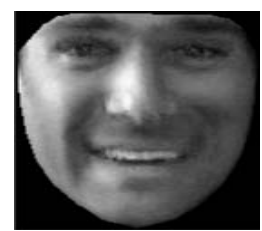

(c) FEAF warp

Fig. 4. Comparison of expression reconstruction
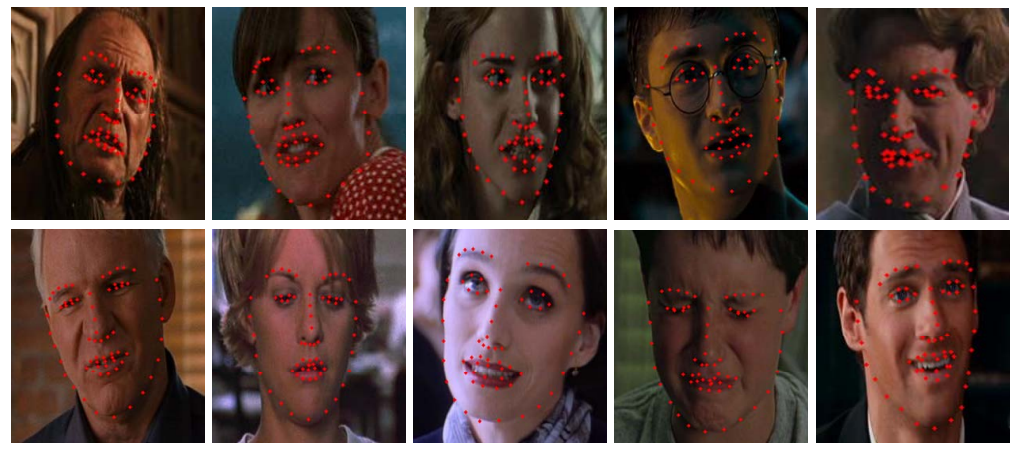

Fig. 5. Facial landmark localization

local block. The problem of facial expression recognition is a 7-class classification task, so RBF kernel based SVM classifier with one-vs-rest strategy is used for multi-class classification.

Table 1 illuminates the comparison with the state-of-the-art approaches for FER in the wild. The result of baseline is obtained from database creators [22]. It neither employs view-invariant approaches nor addresses the problem of discriminative feature learning. So baseline shows inferior performance than most view-invariant FER methods. In [6], the latest approach of view-normalization model is presented. It achieves relatively high accuracy in Angry, Disgust and Sadness. Nevertheless, its recognition rates of Neutral and Surprise are extremely 
Table 1. Recognition rate (\%) of different methods on SFEW databse

\begin{tabular}{|c|c|c|c|c|c|c|c|c|}
\hline & Angry & Disgust & Fear & Happy & Neutral & Sadness & Surprise & Total \\
\hline Baseline & 23.00 & 13.00 & 13.90 & 29.00 & 23.00 & 17.00 & 13.50 & 18.90 \\
\hline$[6]$ & $\mathbf{2 5 . 8 9}$ & $\mathbf{2 8 . 2 4}$ & 17.17 & 42.98 & 14.00 & $\mathbf{3 3 . 3 3}$ & 10.99 & 24.70 \\
\hline$[24]$ & 24.11 & 14.12 & 20.20 & 50.00 & 23.00 & 23.23 & 21.98 & 26.14 \\
\hline FEAF & 23.21 & 18.82 & $\mathbf{2 3 . 2 3}$ & $\mathbf{5 0 . 8 8}$ & $\mathbf{4 0 . 0 0}$ & 26.26 & $\mathbf{2 9 . 6 7}$ & $\mathbf{3 0 . 8 6}$ \\
\hline
\end{tabular}

poor, even lower than random guess. This situation reveals the unstable performance of [6]. As previously discussed, view-normalization approaches needs large amount of training data to ensure accuracy. Considering SFEW is a small-scale database, the result of this method is consequently unstable. In [24], a stateof-the-art approach of dictionary-learning model is displayed and its result is a little better than [6]. Dictionary-learning methods, thus, are more stable than view-based methods for small sample experiment. The performance of FEAF can be summarized as below:

- FEAF outperforms all the other methods in terms of the overall recognition rate.

- FEAF achieves a considerable improvement in Fear, happy, Neutral and Surprise. Especially for the Neutral, our method achieves significantly higher accuracy than others.

- Unlike [6] which is bias against small sample data analysis, there is no obvious bias of FEAF.

- FEAF shows superior performance when compared with the state-of-the-art view-invariant FER.

\section{Conclusions}

In this paper, to the best of our knowledge, we present the first facial expressionaware face frontalization (FEAF) method for FER in the wild. FEAF includes three main step: multi-template design, template matching and improved Robust Statistical face Frontalization (RSF). The proposed method can successfully achieve generic face frontalization in 2D. The detailed information of facial expression, meanwhile, is maintained. The experimental validation shows that FEAF outperforms the state-of-the-art view-invariant FER methods. In the future, we are expected to further improve the accuracy of FEAF by developing a more effective template matching technique.

Acknowledgement. This work was supported by EU seventh framework programme under grant agreement No. 611391, DREAM, and the EPSRC project, 4D Facial Sensing and Modelling (EP/N025849/1). 


\section{References}

1. Valstar, M., Jiang, B., Mehu, M., Pantic, M., Scherer, K.: The first facial expression recognition and analysis challenge. In: IEEE International Conference and Workshops on Automatic Face and Gesture Recognition. (2011) 921-926

2. Sariyanidi, E., Gunes, H., Cavallaro, A.: Automatic analysis of facial affect: A survey of registration, representation and recognition. IEEE Transaction on Pattern Analysis and Machine Intelligence 37 (2015) 1113-1133

3. Zeng, Z., Pantic, M., Roisman, G.I., Huang, T.S.: A survey of affect recognition methods: Audio, visual, and spontaneous expressions. IEEE Transaction on Pattern Analysis and Machine Intelligence 31 (2009) 39-58

4. Moore, S., Bowden, R.: Local binary patterns for multi-view facial expression recognition. Computer Vision and Image Understanding 115 (2011) 541-558

5. Hesse, N., Gehrig, T., Gao, H., Ekenel, H.K.: Multi-view facial expression recognition using local appearance features. International Journal of Computer Vision 83 (2011) 178-194

6. Eleftheriadis, S., Rudovic, O., Pantic, M.: Discriminative shared gaussian processes for multiview and view-invariant facial expression recognition. IEEE Transaction on Image Processing 24 (2015) 189-204

7. Rudovic, O., Pantic, M., Patras, I.: Coupled gaussian processes for pose-invariant facial expression recognition. IEEE Transaction on Pattern Analysis and Machine Intelligence 35 (2013) 1357-1369

8. Tariq, U., Yang, J., Huang, T.S.: Multi-view facial expression recognition analysis with generic sparse coding feature. In: Procceedings of European Conference on Computer Vision. (2012) 578-588

9. Tariq, U., Yang, J., Huang, T.S.: Supervised super-vector encoding for facial expression recognition. Pattern Recognition Letters 46 (2014) 89-95

10. Lowe, D.G.: Object recognition from local scale-invariant features. In: Proceedings of 7th IEEE International Conference on Computer Vision. Volume 2. (1999) 11501157

11. Jeni, L.A., Cohn, J.F., Kanade, T.: Dense 3d face alignment from $2 \mathrm{~d}$ videos in real-time. In: IEEE International Conference and Workshops on Automatic Face and Gesture Recognition. (2015) 1-8

12. J. Roth, Y.T., Liu, X.: Unconstrained 3d face reconstruction. In: IEEE International Conference Computer Vision Workshops. (2015) 2606-2615

13. Hassner, T., Harel, S., Paz, E., Enbar, R.: Effective face frontalization in unconstrained images. In: IEEE Conference on Computer Vision and Pattern Recognition. (2015) 4295-4304

14. Ho, H.T., Chellappa, R.: Pose-invariant face recognition using markov random fields. IEEE Transaction on Image Processing 22 (2013) 1573-1584

15. Sagonas, C., Panagakis, Y., Zafeiriou, S., Pantic, M.: Robust statistical face frontalization. In: Proceedings of IEEE International Conference on Computer Vision. (2015) 3871-3879

16. Taheri, S., Qiu, Q., Chellappa, R.: Structure-preserving sparse decomposition for facial expression analysis. IEEE Transaction on Image Processing 23 (2014) 35903603

17. Xiong, X., la Torre, F.D.: Supervised descent method and its applications to face alignment. In: IEEE Conference on Computer Vision and Pattern Recognition. (2013) 532-539 
18. Xue, M., Liu, W., Li, L.: Person-independent facial expression recognition via hierarchical classification. In: IEEE International Conference on Intelligent Sensors, Sensor Networks and Information Processing. (2013) 449-454

19. Zhang, Z., Ganesh, A., Liang, X., Ma, Y.: Tilt: Transform invariant low-rank textures. International Journal of Computer Vision 99 (2012) 1-24

20. Cootes, T.F., Taylor, C.J., Cooper, D.H., Graham, J.: Active shape model-their training and application. Computer Vision and Image Understanding 61 (1995) $38-59$

21. Metthews, I., Baker, S.: Active appearance model revisited. International Journal of Computer Vision 60 (2004) 135-164

22. Dhall, A., Goecke, R., Lucey, S., Gedeon, T.: Static facial expression analysis in tough conditions: Data evaluation protocol and benchmark. In: IEEE International Conference Computer Vision Workshops. (2011) 2106-2112

23. Huang, D., Shan, C., Ardabilian, M., Wang, Y., Chen, L.: Local binary patterns and its application to facial image analysis: A survey. IEEE Transactions on Systems, Man, and Cybernetics, Part C: Aplications and Reviews 41 (2011) 765-781

24. Liu, M., Li, S., Chen, X.: Au-aware deep networks for facial expression recognition. In: IEEE International Conference and Workshops on Automatic Face and Gesture Recognition. (2013) 1-6 